\title{
Psicooncología
}

ISSN: $1696-7240$

https://dx.doi.org/10.5209/psic.65595

\section{Herramienta de medición de la satisfacción de pacientes oncológicos en tratamiento con quimioterapia en régimen ambulatorio SAMBO-14}

\author{
Juana Ma Sepúlveda-Sánchez'; Francisco Rivas-Ruiz²; Antonio Rueda Dominguez ${ }^{3}$; Silvia \\ Aguilar Carmona ${ }^{4}$; Concepción Ruiz Hernandez ${ }^{5}$, José Carlos Canca-Sánchez ${ }^{6}$.
}

\section{Recibido: 26 de julio de 2018 / Aceptado. 1 de junio de 2019}

Resumen: Objetivo: Diseño y validación de un instrumento de medida de la satisfacción manifestada por pacientes en tratamiento oncológico en régimen ambulatorio en Hospital de Día. Método: Estudio transversal descriptivo de periodo mediante la administración de un cuestionario a una muestra de 148 pacientes oncológicos atendidos en régimen ambulatorio en el Hospital Costa del Sol, Marbella. Se analizaron la validez, consistencia interna, y la reproducibilidad de los items del instrumento. Resultados: La correlación ítem-escala mostró coeficientes que superaban el umbral de adecuación de 0,30. El análisis factorial de los componentes determinó un adecuado ajuste dado un valor de KMO de 0,91 y una $p<0,001$ en el test de esfericidad de Barlett. El análisis de consistencia interna determinó coeficientes de alfa de Cronbach $>0,70$. El retest determinó porcentajes de acuerdo absoluto superiores al $90 \%$ en todos los ítems, y valores de kappa puntuales que oscilaban entre 0,52 y 0,93 . Conclusión: Los resultados obtenidos permiten determinar que a priori el cuestionario es un instrumento fiable y válido para su utilización en lengua castellana.

Palabras clave: Neoplasias, satisfacción del paciente, encuestas y cuestionarios, validez social de la investigación, atención ambulatoria.

\section{[en] SAMBO-14: A tool for measuring satisfaction of cancer patients receiving ambulatory chemotherapy}

\begin{abstract}
Purpose: Design and validation of an instrument measuring the satisfaction of patients receiving ambulatory cancer therapy in a day hospital. Methods: Descriptive cross-sectional study involving the administration of a questionnaire to a sample of 148 cancer patients seen in an ambulatory
\end{abstract}

1 Servicio de Oncología. Agencia Pública Empresarial Costa del Sol. 29603. Marbella. España.

E-mail: juanamss@hcs.es

2 Unidad de Investigación. Red de Investigación en Servicios de Salud en Enfermedades Crónicas. REDISSEC. Agencia Pública Empresarial Costa del Sol. 29603. Marbella. España.

E-mail: frivasr@hcs.es

3 Dirección Servicio de Oncología. Agencia Pública Empresarial Costa del Sol. 29603. Marbella. España.

E-mail: rueda_dominguez@gmail.com

4 Servicio de Oncología. Agencia Pública Empresarial Costa del Sol. 29603. Marbella. España. España. E-mail:sac79@hotmail.es

5 Servicio de Oncología. Agencia Pública Empresarial Costa del Sol. 29603. Marbella. España. E-mail: coruhe@hotmail.com

6 Dirección de enfermería. Agencia Pública Empresarial Costa del Sol. 20603. Marbella. España.

E-mail: jccanca@hcs.es

* Dirección de correspondencia: Juana Ma Sepúlveda Sánchez. Servicio de Oncología. Agencia Publica Empresarial Costa del Sol. Autovía A-7, Km 187. 29603 Marbella, Málaga (Spain). E-mail: juanamss@hcs.es 
setting at Hospital Costa del Sol, Marbella (Spain). The validity, internal consistency and reproducibility of the instrument's items were analysed. Results: In the item-scale correlation, the coefficients obtained were above the adequacy threshold of 0.30 . Results showed that the principal components factor analysis was appropriate based on a KMO value of 0.91 and a $p<0.001$ for Bartlett's sphericity test. The internal consistency analysis established Cronbach's alpha coefficients $>0.70$. A test-retest analysis revealed absolute percentage agreements greater than $90 \%$ for all items and kappa point estimates ranging from 0.52 to 0.93 . Conclusions: The results obtained show that the questionnaire is, a priori, a reliable and valid instrument for use in Spanish language.

Keywords: Neoplasms, patient satisfaction, surveys and questionnaires, social validity research, ambulatory care.

Sumario. 1. Introducción 2. Método 2.1. Diseño 2.2. Estudio de Validación 3. Análisis estadístico 4. Resultados 5. Discusión 6. Conclusión 7. Referencias bibliográficas

Cómo citar: Sepúlveda-Sánchez JM, Rivas-Ruiz F, Rueda Dominguez A, Aguilar Carmona S, Ruiz Hernandez C, Canca-Sánchez JC. Herramienta de medición de la satisfacción de pacientes oncológicos en tratamiento con quimioterapia en régimen ambulatorio SAMBO-14.Psicooncología 2019; 16: 345356. doi: $10.5209 /$ psic. 65595

\section{Introducción}

Las Unidades de Hospital de Día se definen según el Real Decreto 1277/2003 ${ }^{(1)}$ por el que se establecen las bases generales sobre autorización de centros, servicios y establecimientos sanitarios como "la unidad asistencial donde bajo la supervisión ó indicación de un médico especialista, se lleva a cabo el tratamiento o los cuidados de enfermos que deben ser sometidos a métodos de diagnóstico y tratamiento que requieren durante unas horas atención continuada médica o de enfermería, pero no el internamiento en el hospital".

Esta modalidad de atención que supone una alternativa a la hospitalización, tiene su origen en la década de los 70 en Estados Unidos con la especialidad de hematología para dar soporte a procedimientos diagnósticos y la administración de terapias diversas. En la actualidad la atención ambulatoria se ha hecho extensiva a otras especialidades médicas, quirúrgicas y pediátricas mediante la implantación en los centros sanitarios de unidades polivalentes de Hospitales de Día. Estos servicios han mejorado la accesibilidad y el confort de los pacientes y han generado una mejora de la eficiencia asistencial liberando camas hospitalarias mediante la reducción de ingresos, ingresos no adecuados y reingresos precoces en las unidades implicadas ${ }^{(2)}$.

En cuanto al empleo de esta modalidad para la atención del paciente con cáncer, los estudios muestran que la satisfacción de los pacientes tratados en el hospital de día y las consultas externas de oncología es alta ${ }^{(3,4)}$. Destacan que aspectos tales como el "sentido de la normalidad" y "ausencia del rol de enfermo" contribuyen al mantenimiento de su calidad de $v_{i d a}{ }^{(5)}$. La utilidad de la información recibida, la preferencia del desplazamiento que supone la atención en el hospital de día con respecto al internamiento, y la posibilidad de continuar los cuidados en el domicilio son otros aspectos valorados positivamente ${ }^{(6)}$. Además, la incorporación de nuevas formas de atención como el seguimiento telefónico ${ }^{(7)}$ y la incorporación de la figura del farmacéutico ${ }^{(8)}$ en estas unidades también han sido identificadas como medidas positivas para el control de síntomas y toxicidades y la mejora de la educación del paciente en las estrategias de autocuidado. 
No obstante, y aunque el grado de insatisfacción parece estar más relacionado con el estado de salud global del paciente ${ }^{(9)}$, con factores sociodemográficos como la corta edad y el estado civil, y con el tipo de tumor ${ }^{(3)}$, existen estudios que muestran dimensiones relacionadas con la organización del servicio que inciden negativamente en la calidad de la atención recibida, y dominios de la información proporcionadas al paciente y su familia no satisfechas ${ }^{(10)}$. Aspectos relacionados con los tiempos de espera ${ }^{(11)}$, el "sentido de camaradería" con otros pacientes y el sentimiento de deshumanización ${ }^{(12)}$, la necesidad de apoyo psicoemocional y la continuidad al alta ${ }^{(6)}$ requieren un mayor análisis y seguimiento para garantizar la calidad en la atención recibida.

En cuanto a los instrumentos de medida de la calidad sanitaria percibida por los pacientes con cáncer y el grado de satisfacción, se han publicado revisiones sistemáticas que concluyen que las herramientas disponibles son susceptibles de mejora. En 2014, Tzelepis et al. analizaron las herramientas de medida de la calidad de la atención expresada por los usuarios en base a las dimensiones que el Instituto de Medicina reconoció como esenciales en 2001: eficacia, oportunidad, seguridad, eficiencia, equidad y atención centrada en el paciente ${ }^{(13)}$, concluyendo que solo uno de los 21 instrumentos hallados incluía preguntas relacionadas con estas seis dimensiones, pero que requería de pruebas psicométricas adicionales antes de poder extraer conclusiones definitivas sobre su confiabilidad y validez ${ }^{(14)}$. Por otro lado, la revisión sistemática llevada a cabo por Brédart en 2015 sobre instrumentos de medida del grado de satisfacción de pacientes con cáncer en tratamiento ambulatorio, apuntó que los estudios proporcionan información psicométrica insuficiente, y que, aunque la mayoría de las estimaciones de consistencia interna son satisfactorias, pocos informan de la evaluación test-retest. Así mismo, establecen que la transición, la accesibilidad y la participación de la familia son las dimensiones menos evaluadas a pesar de su relevancia en la oncología ambulatoria ${ }^{(15)}$.

Dada las conclusiones de las últimas revisiones publicadas y la necesidad de disponer de una herramienta adaptada a la especificidad y las características de nuestro servicio, nos propusimos diseñar y validar un instrumento específico de medida de la calidad de la atención y la satisfacción percibida por los pacientes en tratamiento ambulatorio con quimioterapia en el Hospital de Día Oncohematológico de la Agencia Sanitaria Costa de Marbella, Málaga.

\section{Método}

Estudio transversal descriptivo de periodo realizado entre abril y noviembre de 2017 con usuarios del Hospital de Día Oncohematológico de la Agencia Sanitaria Costa del Sol. El criterio de inclusión fue ser paciente mayor de 18 años de la especialidad de Oncología o Hematología y encontrarse recibiendo tratamiento ambulatorio en el centro desde al menos dos meses previos al momento del estudio. Se excluyeron pacientes con dificultades cognitivas, barrera idiomática y aquellos que se negaron a colaborar. La muestra se seleccionó de forma consecutiva entre los pacientes que cumplían criterios de inclusión durante los 8 meses en los que el estudio se llevó a cabo. Se recogieron un total de 148 encuestas y hubo 2 rechazos a participar. El cuestionario fue administrado por enfermeras del Hospital de Día previa firma del consentimiento informado por escrito y tras la finalización del tratamiento. 


\subsection{Diseño}

La encuesta fue diseñada a partir de los resultados de un grupo nominal dirigido por miembros del equipo de oncohematología en el que participaron 6 pacientes en tratamiento oncológico por un periodo superior a 6 meses, y 2 cuidadores. El estudio se llevó a cabo en Julio de 2016 una vez obtenido el consentimiento por escrito de los participantes. Tras consultar la bibliografía se identificaron una serie de dimensiones a explorar (Tabla 1). La dinámica se desarrolló en tres fases: una primera fase para la exposición del objetivo, finalidad y metodología, una segunda fase de generación de ideas mediante la exposición de preguntas y respuestas anónimas y una fase final de priorización mediante la agrupación de ideas, nominación del grupo, y puntuación de la prioridad. De esta manera se obtuvo una de lista de los aspectos considerados claves para proporcionar una atención ambulatoria de calidad, que posteriormente fueron redactados en forma de preguntas y que sirvieron de base para elaborar un instrumento de medida formado por 14 cuestiones con una escala Likert de medición de 5 ítems. Se añadieron dos ítems más para medir la satisfacción de los pacientes hacia los profesionales sanitarios y servicio de hospital de día a partir de una escala de 1 a 10 puntos.

Tabla 1. Dimensiones exploradas y cuestiones planteadas. Técnica de grupo nominal.

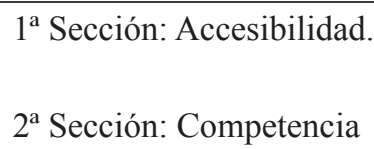

$3^{\text {a }}$ Sección: Continuidad Asistencial

$4^{a}$ Sección. Comprensión-lenguaje

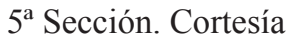

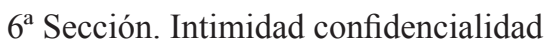

$7^{a}$ Sección. Acompañamiento
¿Qué ventajas e inconvenientes ven en recibir el tratamiento en una Unidad de Día?

¿Qué aspectos considera que hay que mejorar ó incluir como parte de la atención que ofrecemos?

¿Qué aspectos considera débiles en su relación con el equipo que le atiende?

¿Qué aspectos sanitarios consideran que no han sido totalmente cubiertos en los periodos interciclos?

¿Cómo cree que se podría mejorar el proceso de atención desde la sospecha del diagnóstico hasta que recibe el tratamiento?

¿Qué problemas o dificultades ha encontrado en la información ofrecida por el equipo de profesionales que le atiende?

¿Qué mejoraría con respecto al trato recibido en el servicio de Hospital de Día?

¿Qué dificultades encuentra a la hora de respetar su derecho a la intimidad y confidencialidad en el servicio?

¿Qué dificultades encuentra a la hora de facilitar el acompañamiento de su familiar? 


\subsection{Estudio de Validación}

Para evaluar la validez y fiabilidad del nuevo cuestionario se plantearon las siguientes fases de validación:

1. Se contrastó la validez aparente del cuestionario en una muestra de 10 profesionales del servicio que no participaron en el grupo nominal, realizando modificaciones en la redacción de 5 de las 14 cuestiones planteadas para mejorar el grado de comprensión de la dimensión explorada.

2. Se realizó un pretest para determinar su ajuste en 30 pacientes. Se comprobó la comprensibilidad de los diferentes ítems y se adaptó la formulación de los mismos.

3. Se valoró la validez de constructo y fiabilidad-homogeneidad una vez administrado a la muestra seleccionada (148 pacientes).

4. Finalmente se valoró su grado de fiabilidad-estabilidad en una submuestra de 30 pacientes y con una media de 30 días entre evaluaciones.

\section{Análisis estadístico}

Se realizó un análisis descriptivo de las características demográficas y tiempo en tratamiento de la muestra de pacientes de validación (148 pacientes). En la misma muestra, se evaluó la frecuencia de endose para todos los ítems de satisfacción con respuesta de escala Likert de 5 puntos, y se realizó la correlación ítem-escala, considerándose a estudio aquellos ítems con coeficiente de correlación (CC) de Pearson por encima de 0,30 . La validez de concepto se evaluó mediante un análisis factorial exploratorio sobre componentes principales (AFCP), transformando las soluciones con rotación Varimax. La adecuación del análisis factorial se comprobó mediante la medida de Kaiser-Meyer Olkin (KMO) y la prueba de esfericidad de Barlett. Se consideraron aquellos ítems con comunalidades y saturaciones factoriales superiores a 0,50 .

La consistencia interna de los ítems de cada uno de los componentes resultantes se evaluó a partir del coeficiente Alfa de Cronbach, considerándose valores de alfa relevantes los superiores a $0,70^{(16)}$.

Se estimó la estabilidad de los ítems mediante la prueba test-retest, valorando la proporción de acuerdo absoluto (Pa) y el Kappa ponderado por pesos de Cicchetti ${ }^{(17)}$.

Este estudio fue aprobado por el Comité de Ética de Investigación de la Agencia Sanitaria Costa del Sol en Marzo de 2017.

\section{Resultados}

Se recogieron un total de 148 encuestas. El 50,7\% eran mujeres, para una media de 57,9 (DT: 11,8) años. La mediana de tiempo de tratamiento en hospital de día fue de 4 meses (RI: 2 -10,8), con una media de tiempo de tratamiento de 5,7 (DT: 3,1) meses. Los datos descriptivos de la muestra se detallan en la tabla 2. 
Tabla 2. Descriptiva de la población de estudio.

\begin{tabular}{lrcc}
\hline Variables & & Frecuencia & Porcentaje \\
\hline Sexo & & & \\
& Hombre & 73 & 49,3 \\
& Mujer & 75 & 50,7
\end{tabular}

Edad

Media - DT $\quad 57,9 \quad 11,8$

Tiempo en tratamiento en Hospital de Día (meses)
Mediana - RI
4,0
$(2-10,8)$

Tiempo en tratamiento

Media - DT

Satisfacción con los profesionales

Media - DT

9,6

0,8

Satisfacción con el Hospital de Día

Media - DT

9,3

1,1

En la evaluación del porcentaje de cumplimentación de los 14 ítems de la encuesta, para los 12 primeros ítems de la encuesta respondieron la totalidad de los encuestados, mientras que respondieron el $79,1 \%$ y el 81,8 para los 13 y 14 , respectivamente (Tabla 3 ).

Respecto a la distribución de las frecuencias en las categorías de los ítems del cuestionario, para todos los ítems se superó el 70\% de satisfacción (sumando categoría "Satisfecho" y "Muy Satisfecho), obteniéndose cifras superiores al 90\% de satisfacción para ítems $3,8,10,11,12$ y 14 . Por otra parte, en la correlación ítemescala, todos los coeficientes superaban el umbral de adecuación de 0,30 , siendo el más bajo hallado para el ítem $13(0,47)$ y el más alto el del ítem $7(0,76)$, por lo que todos los ítems eran adecuados para el posterior análisis factorial.

La satisfacción de los pacientes evaluada con escala de 1 a 10 puntos, fue de 9,6 (DT: 0,8) para los profesionales sanitarios, y de 9,3 (DT: 1,1) para el Hospital de Día.

En el análisis factorial de componentes principales realizado mediante 14 ítems con escala Likert, se determinó un adecuado ajuste dado un valor de KMO de 0,91 y con una $\mathrm{p}<0,001$ en el test de esfericidad de Barlett. Las comunalidades de los ítems oscilaron entre 0,49 para ítem 5, y 0,84 para el ítem 1 . Así, se determinaron tres componentes (con saturaciones factoriales que superaban valor 0,5 ) que explicaban cerca del $65 \%$ de la varianza; componente $\mathrm{C} 1$ denominado "intimidad e información durante el proceso", componente $\mathrm{C} 2$ denominado "organización funcional del servicio" y componente C3 "continuidad asistencial". El primer componente identificado (ítems 6, 7, 8, 9, 10, 11, y 12) explica el 48,8\% de la varianza, el segundo componente (ítems $1,2,3,4,5$ ) explica el $8,5 \%$ de la varianza, y el tercer componente (ítems 13 y 14) explica el $7,7 \%$ de la varianza. El análisis de consistencia interna determinó coeficientes de alfa de Cronbach superiores a un valor de adecuación fijado de 0,7 en tres componentes identificados por análisis factorial de componentes principales (Tabla 4). 


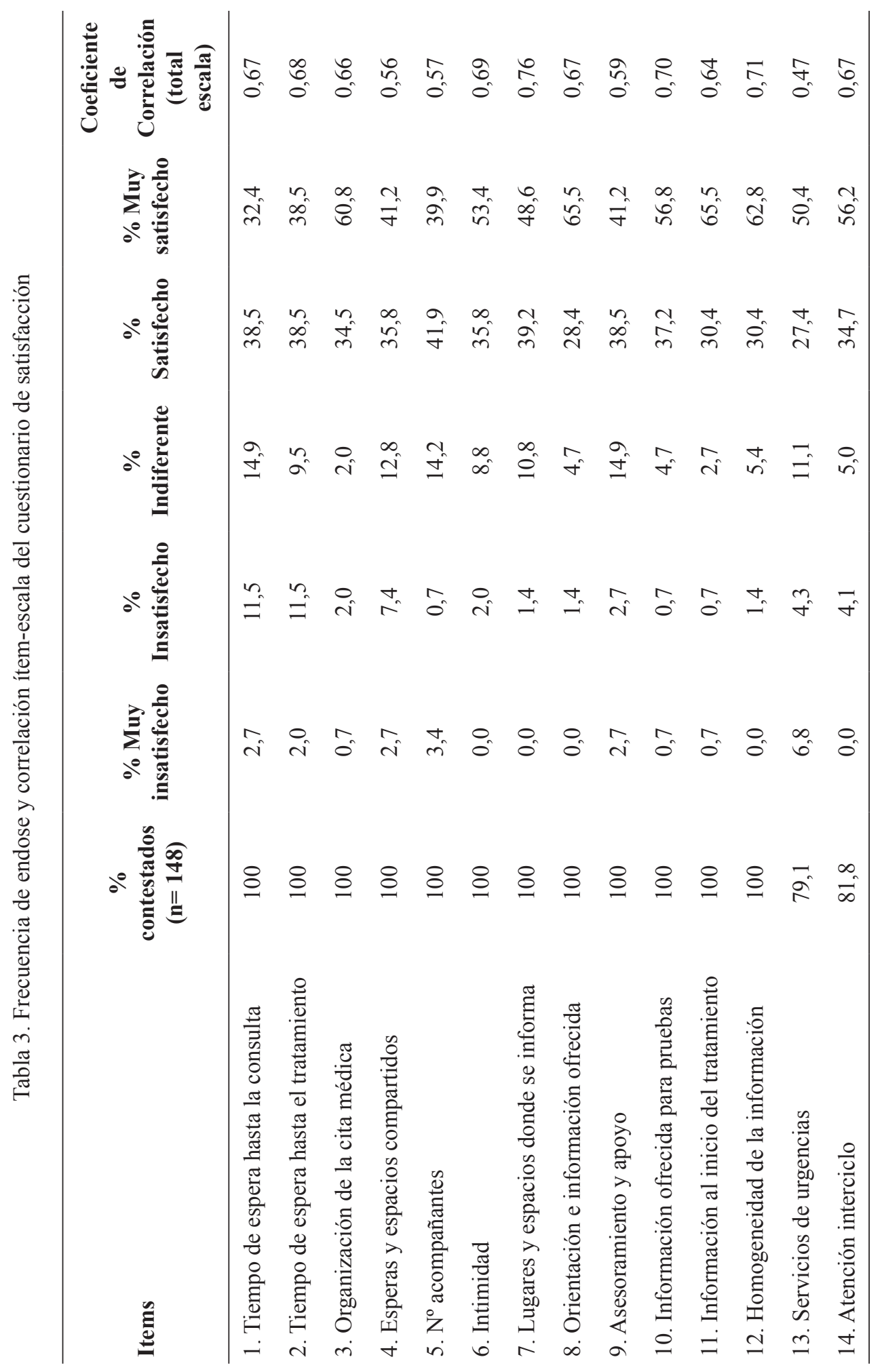




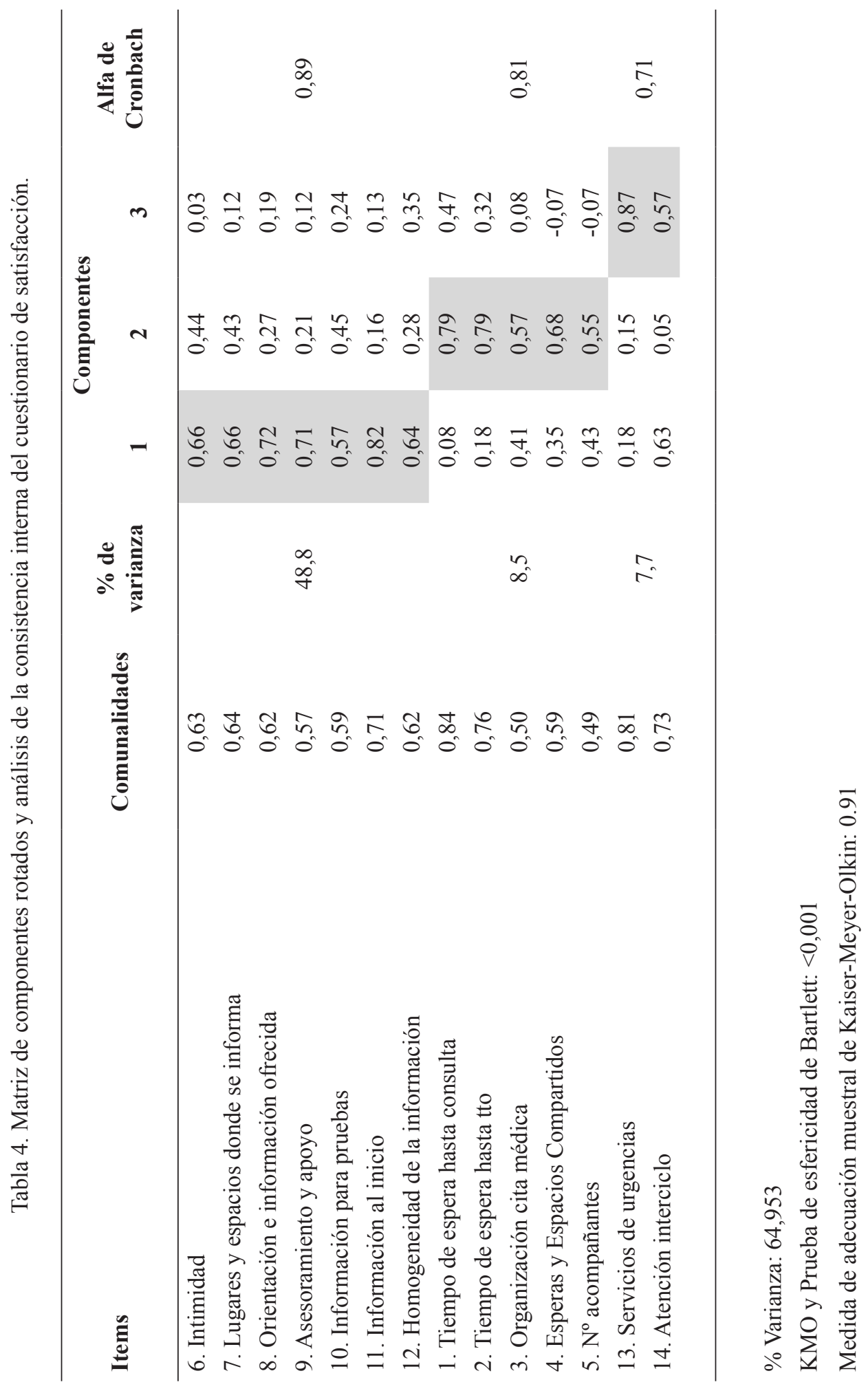


En el test-retest se determinaron porcentajes de acuerdo absoluto superiores al $90 \%$ en todos los ítems, y valores de kappa puntuales que oscilaban entre 0,52 en ítem 8 y 0,93 en ítem 14 (Tabla 5).

Tabla 5. Proporción de acuerdo absoluto y valores kappa de ítems del cuestionario de satisfacción.

\begin{tabular}{lcc}
\hline \multicolumn{1}{c}{ Items } & Pa & Kappa (IC95\%) \\
\hline 1. Tiempo de espera hasta consulta & 91,7 & $0,69(0,52-0,86)$ \\
2. Tiempo de espera hasta el tratamiento & 94,2 & $0,76(0,59-0,93)$ \\
3. Organización cita médica & 94,2 & $0,56(0,30-0,83)$ \\
4. Esperas y Espacios Compartidos & 92,5 & $0,61(0,41-0,81)$ \\
5. $\mathrm{N}^{\mathrm{o}}$ acompañantes & 96,7 & $0,84(0,69-0,99)$ \\
6. Intimidad & 92,5 & $0,55(0,31-0,78)$ \\
7. Lugares y espacios donde se informa & 95,0 & $0,76(0,57-0,94)$ \\
8. Orientación e información ofrecida & 93,3 & $0,52(0,23-0,80)$ \\
9. Asesoramiento y apoyo & 96,7 & $0,84(0,68-0,99)$ \\
10. Información para pruebas & 95,8 & $0,72(0,50-0,94)$ \\
11. Información al inicio & 96,7 & $0,74(0,52-0,95)$ \\
12. Homogeneidad de la información & 94,2 & $0,68(0,46-0,89)$ \\
13. Servicios de urgencias & 93,1 & $0,84(0,68-0,99)$ \\
14. Atención interciclo & 98,2 & $0,93(0,84-1,00)$ \\
Pa: Porcentaje de Acuerdo Absoluto; IC95\%: Intervalo de Confianza al 95\% \\
\hline
\end{tabular}

Tiempo Test-Retest: mediana 30 (RI: 20-55) días

\section{Discusión}

Actualmente existen instrumentos de medida validados y traducidos que miden la calidad de vida durante la terapia del cáncer. El FACIT-TS-P fue validado en 1998 en pacientes con cáncer nativos de habla hispana que vivían en Estados Unidos y Puerto Rico para su uso en ensayos clínicos y práctica clínica, y dirigido al paciente en tratamiento con enfermedades crónicas ${ }^{(18)}$. Más adelante el cuestionario EORTC In-Patsat32 mostró solidez psicométrica para la evaluación del personal sanitario, así como aspectos relativos a la organización y atención sanitaria ${ }^{(19)}$, y posteriormente se adaptó a la versión EORTC Out-Patsat35 para explorar la satisfacción del paciente relacionada con la calidad de vida en Francia en pacientes en tratamiento ambulatoria de quimioterapia y radioterapia en $2006^{(20)}$ (EORTC Out Pastsat35 CT y EORTC Out Pastsat35 RAT respectivamente). En concreto, el EORTC Out Pastsat35 RAT ha sido validado en pacientes españoles en tratamiento con radioterapia mostrando resultados alineados con los del estudio de validación realizado por los autores ${ }^{(21)}$, no obstante 
no se han analizado las propiedades psicométricas del EORTC Out Pastsat35 CT en pacientes españoles en tratamiento ambulatorio de quimioterapia, si bien sólo difiere en las instrucciones que son específicas para cada tipo de tratamiento.

Los cuestionarios de satisfacción de la atención sanitaria en pacientes oncohematológicos en tratamiento ambulatorio son adaptaciones de instrumentos elaborados en otros países, frente a nuestro cuestionario que ha sido elaborado a partir de la información reportada en un estudio cualitativo por nuestros propios pacientes. Es un cuestionario breve en tiempo de administración, autoadministrado $\mathrm{y}$ asequible para cualquier perfil educativo. Su uso y posterior análisis permite identificar medidas de mejora en los servicios ambulatorios oncológicos que dan respuesta a las expectativas de los pacientes.

Los 14 ítems identificados cumplen criterios de adecuación de validez, revelando el análisis factorial de componentes principales tres componentes subyacentes de la satisfacción, "intimidad e información durante el proceso" (componente 1), "organización funcional del servicio" (componente 2) y "continuidad asistencial durante el proceso" (componente 3). En futuros estudios con muestras amplias de pacientes, se reflejarán tanto las puntuaciones obtenidas por componentes, como una puntuación global de satisfacción corregida por los pesos de la varianza obtenida en el análisis factorial.

El análisis test-retest ha mostrado una alta estabilidad de los ítems, tanto en la evaluación del porcentaje de acuerdo absoluto, que arrojó cifras excelentes, como por valores de kappa identificados en su mayoría en umbral "bueno" $(0,61$ a 0,80$)$ o "muy bueno" $(>0,80)^{(22)}$.

Una limitación del estudio es el haber recogido la muestra en un solo centro, además de haberse obtenido un efecto techo en varios ítems de satisfacción con escala Likert, junto con los dos ítems de valoración global, con puntuaciones que rozaban la perfección. Se plantearán futuros estudios multicéntricos para replicar el estudio de evaluación de propiedades psicométricas.

\section{Conclusión}

Hemos desarrollado un cuestionario en español a partir de grupo de expertos para medir la satisfacción del paciente oncológico en Hospital de Día: "Cuestionario de satisfacción de la atención ambulatoria oncológica SAMBO-14". Los resultados obtenidos permiten determinar que el instrumento es válido y fiable para su utilización en lengua castellana.

\section{Referencias bibliográficas}

1. Real Decreto $1277 / 2003$, de 10 de octubre, por el que se establecen las bases generales sobre autorización de centros, servicios y establecimientos sanitarios.

2. Escobar MA, Garcia-Egido AA, Carmona R, Lucas A, Márquez C, Gómez F. Ingresos evitados por el hospital de día polivalente. Rev Clin Esp 2012;212:63-74. doi: 10.1016/j. rce.2011.08.003

3. Arraras JI, Illarramendi JJ, Viudez A, Ibáñez B, Lecumberri MJ, de la Cruz S, et al. Determinants of patient satisfaction with care in a Spanish oncology day hospital and its relationship with quality of life. Psychooncology 2013;22:2454-61. doi: 10.1002/pon.3307 
4. Hjörleifsdóttir E, Hallberg IR, Gunnarsdóttir ED. Satisfaction with care in oncology outpatient clinics: psychometric characteristics of the Icelandic EORTC IN-PATSAT32 version. J Clin Nurs 2010;19:1784-94. doi: 10.1111/j.1365-2702.2009.03095.x

5. McIlfatrick S, Sullivan K, McKenna H, Parahoo K. Patients' experiences of having chemotherapy in a day hospital setting. J Adv Nurs 2007;59:264-73. doi: 10.1111/j.13652648.2007.04324.x

6. Sánchez A, Coll ML, Sáez E, Valls C. Evaluación de un hospital de día: monitorizamos indicadores de funcionamiento, pero ¿qué opinan los usuarios?. Rev Calidad Asistencial 2002; 17:337-349. doi: 10.1016/S1134-282X(02)77527-1.

7. Stacey D, Green E, Ballantyne B, Skrutkowski M, Whynot A, Tardif L, et al. Patient and family experiences with accessing telephone cancer treatment symptom support: a descriptive study. Support Care Cancer 2016;24: 893-901. doi: 10.1007/s00520-015-2859-6

8. Crespo A, Tyszka M. Evaluating the patient-perceived impact of clinical pharmacy services and proactive follow-up care in an ambulatory chemotherapy unit. J Oncol Pharm Pract 2017;23:243-248. doi: 10.1177/1078155216634180

9. Nguyen TV, Bosset JF, Monnier A, Fournier J, Perrin V, Baumann C, Brédart A, Mercier M. Determinants of patient satisfaction in ambulatory oncology: a cross sectional study based on the OUT-PATSAT35 questionnaire. BMC Cancer 2011;11:526. doi: 10.1186/1471-2407-11-526

10. Pinto AC, Ferreira-Santos F, Lago LD, de Azambuja E, Pimentel FL, Piccart-Gebhart M. Information perception, wishes, and satisfaction in ambulatory cancer patients under active treatment: patient-reported outcomes with QLQ-INFO25. Ecancermedicalscience 2014;8:425. doi: 10.3332/ecancer.2014.425

11. Lerebours F, Saltel P, Béthune-Volters A, Nallet G, Bourdat P, Vesin-Etterlen F, et al. Satisfaction of Breast Cancer patients treated by chemotherapy outpatient clinics: Results of the TemporELLES survey. Bull Cancer 2015;102:316-23. doi: 10.1016/j. bulcan.2015.01.011

12. McIlfatrick S, Sullivan K, McKenna H, Parahoo K. Patients' experiences of having chemotherapy in a day hospital setting. J Adv Nurs 2007;59:264-73. doi: 10.1111/j.13652648.2007.04324.x

13. Instituto de Medicina. Cruzando el abismo de calidad: un nuevo sistema de salud para el siglo XXI. Washington DC: Prensa de la Academia Nacional; 2001.

14. Tzelepis F, Rose SK, Sanson-Fisher RW, Clinton-McHarg T, Carey ML, Paul CL. Are we missing the Institute of Medicine's mark? A systematic review of patient-reported outcome measures assessing quality of patient-centred cancer care. BMC Cancer 2014; 25:14-41. doi: 10.1186/1471-2407-14-41.

15. Brédart A, Kop JL, Efficace F, Beaudeau A, Brito T, Dolbeault S, et al. Quality of care in the oncology outpatient setting from patients' perspective: a systematic review of questionnaires' content and psychometric performance. Psychooncology 2015; 24:38294. doi: 10.1002/pon.3661.

16. Nunnally JC. Psychometric Theory, 2nd ed. New York: McGraw-Hill, 1978. p. 86-113.

17. Fleiss JL. Statistical methods for rates and proportions. New York: John Wiley \& Sons, 1981

18. Cella D, Hernandez L, Bonomi AE, Corona M, Vaquero M, Shiomoto G, Baez L. Spanish language translation and initial validation of the functional assessment of cancer therapy quality-of-life instrument. Med Care 1998; 36:1407-18.

19. European Organisation for Research and Treatment of Cancer Quality of Life Group and Quality of Life Unit. An international prospective study of the EORTC cancer in-patient 
satisfaction with care measure (EORTC IN-PATSAT32). Eur J Cancer 2005;41:2120-31. doi:10.1016/j.ejca.2005.04.041

20. Poinsot R, Altmeyer A, Conroy T, Savignoni A, Asselain B, Léonard I, et al. Multisite validation study of questionnaire assessing out-patient satisfaction with care questionnaire in ambulatory chemotherapy or radiotherapy treatment. Bull Cancer 2006;93:315-27.

21. Arraras JI, Rico M, Vila M, Chicata V, Asin G, Martinez M, et al. The EORTC cancer outpatient satisfaction with care questionnaire in ambulatory radiotherapy: EORTC OUTPATSAT35 RT. Validation study for Spanish patients. Psychooncology 2010;19:657-64. doi: 10.1002/pon.1611.

22. Altman DG. Practical statistics for medical research. New York: Chapman and Hall, 1991. 